\title{
Characterizing the turbostratic disorder in COF-5 with NMR crystallography
}

Giovanna M. Pope, ${ }^{1}$ Demetrius A. Vazquez-Molina, ${ }^{2}$ Fernando J. Uribe-Romo, ${ }^{2,}{ }^{*}$ James K.

\author{
Harper ${ }^{1, *}$ \\ ${ }^{1}$ Department of Chemistry and Biochemistry, Brigham Young University, C100 BNSN, Provo, UT, \\ 84602 \\ ${ }^{2}$ Department of Chemistry, University of Central Florida, 4111 Libra Drive, Orlando, FL 32816.
}

\begin{abstract}
Since its initial synthesis in 2005 , COF-5 has been known to have intrinsic disorder in the placement of the 2D layers relative to one another (i.e. turbostratic disorder). Prior studies of have demonstrated that the eclipsed layering found in the space group originally assigned to COF-5 $(P 6 / \mathrm{mmm})$ is inconsistent with energy considerations. Herein it is demonstrated that eclipsed layers are also inconsistent with ${ }^{13} \mathrm{C}$ solid-state NMR data. Crystal structure predictions are made in five alternative space groups and good agreement is obtained in $P 21 / \mathrm{m}, \mathrm{Cmcm}$, and $\mathrm{C} 2 / \mathrm{m}$. We posit that all three space groups are present within the stacked 2D layers and show that this conclusion is consistent with evidence from ${ }^{13} \mathrm{C}$ solid-state NMR linewidths and chemical shifts, powder x-ray diffraction data and energy considerations. An alternative explanation involving a mixture of multiple pure phases is rejected because the observed NMR spectra don't exhibit the characteristic features of such mixed phase materials.
\end{abstract}




\section{Introduction.}

In 2005, Côté et al. introduced a class of porous organic materials described as covalent organic frameworks (COFs) ${ }^{[1]}$ COFs are a unique type of crystalline polymer that self-assembles through the formation of reversible covalent bonds between monomers. These materials exhibit low density, high surface area, permanent porosity and a high degree of long range order. The structures of COFs are considered to be predictable based on the principles of reticular chemistry in which a desired topology can be created by an appropriate selection of starting materials. This level of control provides a high degree of tunability and has led to the creation of a remarkable variety of $2 \mathrm{D}$ and $3 \mathrm{D}$ COFs. ${ }^{[2]}$

The distinctive combination of characteristics exhibited by COFs invites an array of applications that have been vigorously pursued over the past 15 years. ${ }^{[3]}$ For example, the COFs are attractive materials for photonic applications due to the long-range alignment of pores between layers. ${ }^{[4]}$ In one study, photon collection was demonstrated using TP-COF while another achieved photocurrent generation with PPy-COF. ${ }^{[5,6]}$ COFs have also been found to be effective catalysts and numerous catalytic, photocatalytic and electrocatalytic applications have been reported. ${ }^{[7,8,9]}$ A notable example of COF catalysis employs an imine-based COF to achieve $\mathrm{CO}_{2}$ fixation using a process that avoids the typical shortcomings (e.g. metal toxicity and product contamination). ${ }^{[10]}$ Certain COFs have been found to act as catalysts for $\mathrm{C}-\mathrm{C}$ coupling reactions, asymmetric Michael additions, $\mathrm{CO}_{2}$ reduction, and water splitting. ${ }^{[11,12,13,14]}$ The COFs have been demonstrated to be effective chemo-sensory materials (e.g. for chemical threat detection and toxic metal detection), conductive membranes, and drug delivery agents. ${ }^{[15,16,17,18]}$ The COFs have also been used for the storage and separation of several greenhouse gases. ${ }^{[19,20]}$ 
The first COFs to be synthesized were designated COF-1 and COF-5. ${ }^{[1]}$ Both 2D frameworks consist of monomers connected through boronate ester linkages and composed entirely of the light elements $\mathrm{B}, \mathrm{C}, \mathrm{O}$, and $\mathrm{H}$. The characteristic topology of these COFs consists of stacked 2D sheets with interlayer interactions dominated by pi-pi stacking. ${ }^{[21]}$ The initial structural studies of COF-1 and COF-5 considered two stacking arrangements: staggered and eclipsed ${ }^{[1]}$ (Figure 1). Hundreds of COFs have now been synthesized with the vast majority reported to crystallize with eclipsed layers. ${ }^{[21]}$
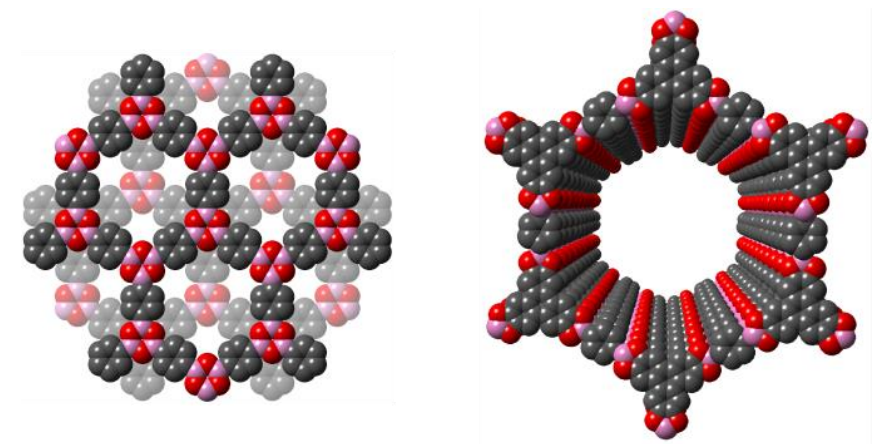

Figure 1. An illustration COF structures that pack with layers that are staggered (COF-1, left) and eclipsed (COF-5, right).

A complication to the proposed eclipsed stacking arrangement of COF-5 was noted in Côtés original report ${ }^{[1]}$ and involved disorder within the stacking of the $2 D$ sheets (i.e. turbostratic disorder). This deviation from a single pure crystalline phase was identified from the unexpected broadening of the (100) and (001) PXRD lines ${ }^{[1]}$ which correspond, respectively, to in-plane and out-of-plane disorder within the 2D layers. This observation led to the consideration of additional stacking possibilities for 2D COFs. ${ }^{[21,22,23,24]}$ In 2011, Lukose et. al. simulated various in-plane offsets between the 2D layers in COF-1, COF-5, COF- 6 and COF-8. ${ }^{[21]}$ For each structure, the electrostatic potential energy and energy of formation were determined. This study revealed that these COFs prefer an offset of $\sim 1.4 \AA$ from the eclipsed stacking. The simulated powder x-ray 
diffraction (PXRD) patterns for the offset structures are fairly insensitive to this offset and thus remain consistent with experimental PXRD data. In a related 2012 study, Koo et. al. further explored the nature of COF stacking by computing potential energy surfaces for $332 \mathrm{D} \mathrm{COFs.}{ }^{[23]}$ These surfaces considered the effects of pi-pi stacking and Coulombic interactions and concluded that none of the 33 frameworks assume a perfectly eclipsed conformation. Instead, these materials adopt offsets ranging from 1.5 to $2.8 \AA$. . In the specific case of COF-5, a $1.6 \AA$ lateral offset was predicted, in good agreement with the 1.4 A offset proposed by Lukose.

The occurrence of offsets in stacked 2D COFs, including COF-5, is significant because it can result in a change in space group designation. Because certain physical properties of a material are dictated by the space group, such a change can have far reaching implications. ${ }^{[25,26]}$ Unfortunately, such small structural changes in COFs are found to only weakly influence the PXRD pattern (vida infra) and alternative analytical methods are needed to assign space group. Solidstate NMR (SSNMR) provides an attractive alternative that is highly sensitive to the local structure including molecular conformation, ${ }^{[27,28]}$ relative stereochemistry, ${ }^{[28,29]}$ number of molecules in the asymmetric unit, ${ }^{[30]}$ hydrogen tunneling ${ }^{[31,32,33]}$ etc. Recent studies have also demonstrated that SSNMR studies can even assign space group in some materials. ${ }^{[34,35]}$ In this report, the influence of turbostratic disorder on the space group assignment for COF-5 is explored using ${ }^{13} \mathrm{C}$ chemical shift tensor principal values. These data consist of three shifts per ${ }^{13} \mathrm{C}$ site, denoted as $\delta_{11}, \delta_{22}$, and $\delta_{33}$. The principal values represent shielding in the three orthogonal directions around each ${ }^{13} \mathrm{C}$ site and have been demonstrated to be highly sensitive to crystal structure. ${ }^{[34,35]}$

Because NMR shift tensor data are primarily sensitive to local structure within a few $\AA$ of a given site, lattice structure is usually difficult to obtain solely from NMR. However, crystal 
structure can be sometimes be obtained if NMR data is combined with crystal structure prediction (CSP) methods. ${ }^{[34,35]}$ The CSP process generates a group of candidate structures in various space groups and numerous approaches have now been evaluated. ${ }^{[36]}$ Because COF-5 is rigid and has layers composed of extended 2D sheets, the possible variations of the lattice structure are limited to different arrangements of the $2 \mathrm{D}$ sheets with respect to one another. This restriction means that a CSP process that considers variations in the packing arrangements between the 2D layers is sufficient to capture the feasible space group variations. Of equal importance is the fact that the original PXRD study of COF-5 identified the directions in which the turbostratic disorder occurs with the most feasible displacement being in the plane parallel to a 2D sheet. Variations in the distance between the sheets are also known to occur from the broadening of the (001) PXRD peak, but because the distance between sheets ( $3.52 \AA)$ is already close to the sum of the van der Waal radius for two carbons (3.40 $)$ ), much less out-of-plane variation is possible. An illustration of the possible candidates that can be created by CSP is illustrated in Figure 2. All candidates include offsets in directions parallel to the 2D sheets. This CSP process has been employed in other materials and found to be effective. ${ }^{[37,38]}$ Herein, a reevaluation of the crystal structure of COF-5 was performed by simulating offsets that correspond to the space groups $\mathrm{P} 31 \mathrm{~m}, \mathrm{P} 21 / \mathrm{m}, \mathrm{C} 2 / \mathrm{m}$ and $\mathrm{Cmcm}$. To generate candidate structures, the eclipsed model predicted in the original PXRD study, ${ }^{[1]} P 6 / \mathrm{mmm}$, served as an initial structure. By applying small offsets to one layer with respect to a second layer in the directions shown in Figure 2, 52 candidate structures were generated. A more detailed description of this process in provided in Experimental. 

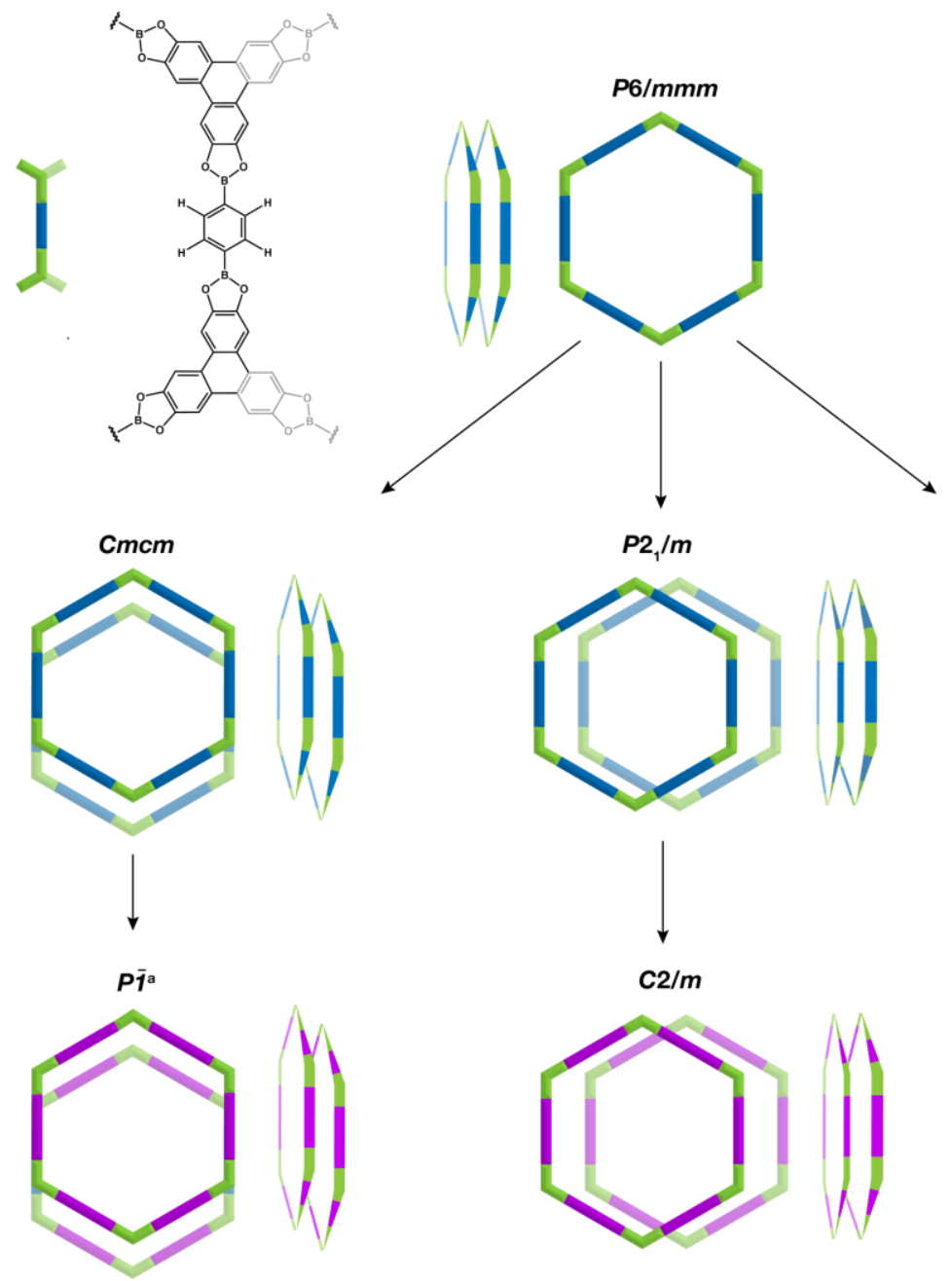

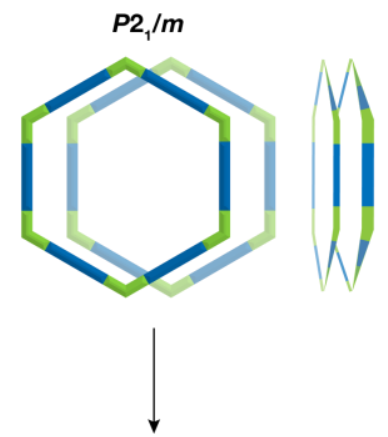

$C 2 / m$

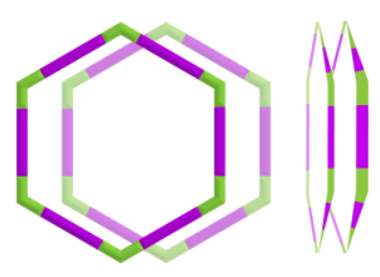

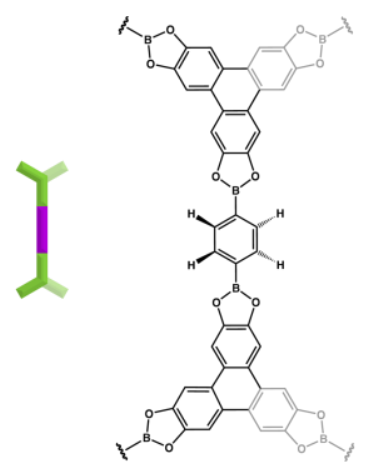
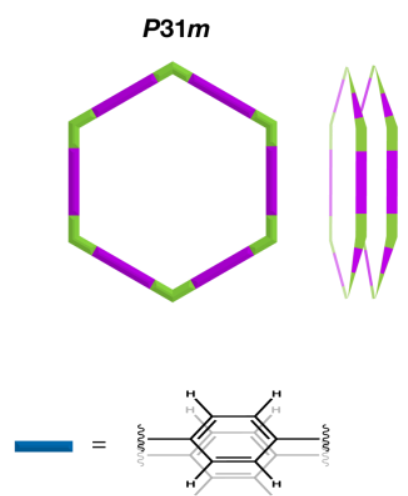

Hydrogen atoms lie in the ab plane. Rings in adjacent layers are parallel.

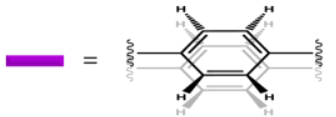

Hydrogens allowed to twist out of the ab plane. Rings in adjacent layers remain parallel.

aNot considered due to very low symmetry.

Figure 2. An illustration of the space groups created by in-plane displacement of the 2D sheets relative to one another in the directions shown. Structures in the $P 6 / \mathrm{mmm}, C_{m c m}, P 2{ }_{1} / \mathrm{m}, \mathrm{P} 31 \mathrm{~m}$ and $\mathrm{C} 2 / \mathrm{m}$ space groups were evaluated herein.

The ${ }^{13} \mathrm{C}$ NMR spectrum of COF-5, shows four unique resonances. A fifth ${ }^{13} \mathrm{C}$ site occurs as a near degenerate line near C4 that can be identified using an interrupted decoupling experiment. ${ }^{[39,40]}$ The ${ }^{13} \mathrm{C}$ shift tensor data for these five sites in COF- 5 was obtained using the FIREMAT experiment. ${ }^{[41]}$ The FIREMAT spectrum obtained for COF-5 is illustrated in Figure 3. Partial shift assignments were obtained from an interrupted ${ }^{1} \mathrm{H}$ decoupling experiment which confirmed the presence of two $\mathrm{CH}$ and three quaternary carbons. The quaternary ${ }^{13} \mathrm{C} \mathrm{C} 5$ 
resonance at $127.5 \mathrm{ppm}$ exhibits a low signal intensity because it represents two carbons that are degenerate due to symmetry. In contrast, all other resonances represent four or six degenerate sites and thus exhibit peak intensities significantly larger than $\mathrm{C} 5$. The intensity of $\mathrm{C} 5$ is further reduced due to its bonding to a quadrupolar nuclei (i.e. boron).

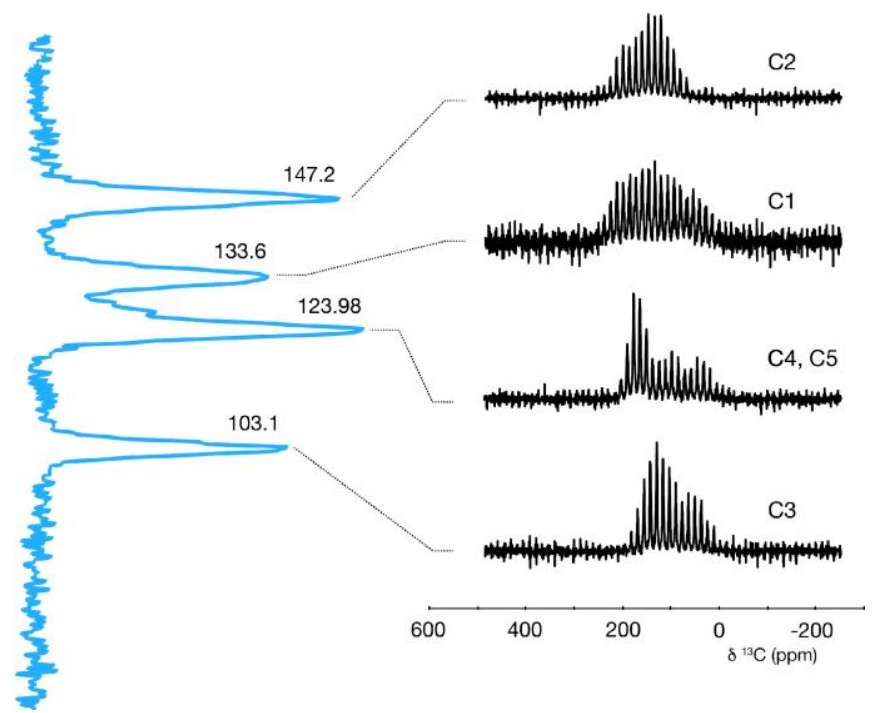

Figure 3. The FIREMAT spectrum of COF-5. 


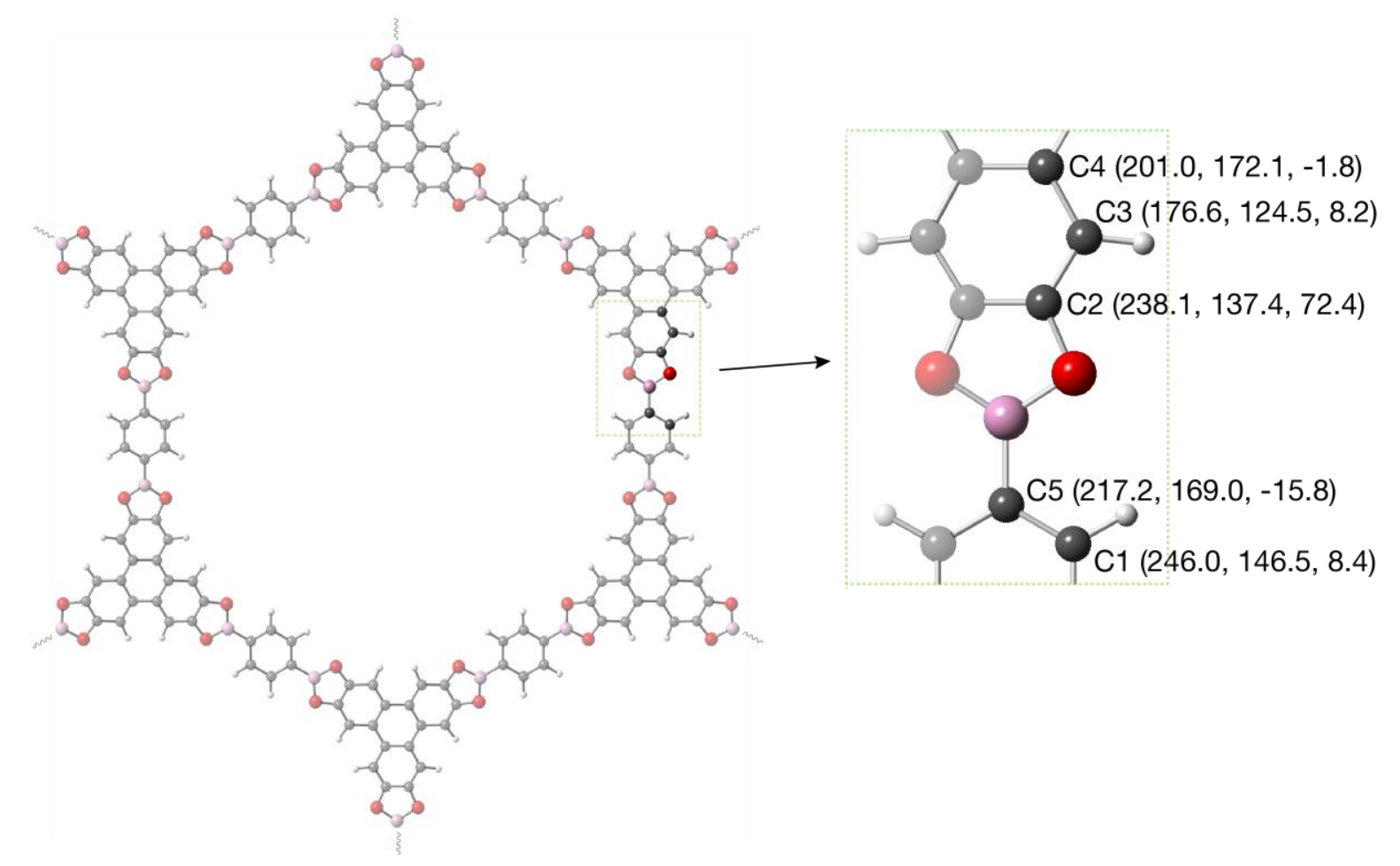

Figure 4. An illustration of a pore from a single 2D sheet within the structure of COF-5. The crystallographic asymmetric unit is shown at the right and results in five unique ${ }^{13} \mathrm{C}$ isotropic resonances. Measured chemical shift tensor principal values for each ${ }^{13} \mathrm{C}$ site are shown.

The ${ }^{13} \mathrm{C}$ shifts were assigned to specific positions by comparing experimental and theoretical shift tensors compute with lattice-including DFT methods. All possible arrangements of computed and theoretical tensors were compared and the arrangement giving the best correlation coefficient was taken as the correct assignment. In each of the 52 candidate structures considered, the same set of shift assignments gave the best-fit and each was statistically preferred over the second-best arrangement. Shift assignments are summarized in Table 1. 
Table 1. The ${ }^{13} \mathrm{C}$ shift assignments for COF-5 and the corresponding chemical shift tensor principal values.

\begin{tabular}{cccccc}
\multicolumn{7}{c}{ Carbon } \\
Position & type & $\delta_{\text {iso. }}$ & $\delta_{11}$ & $\delta_{22}$ & $\delta_{33}$ \\
\hline 1 & $\mathrm{CH}$ & 133.6 & 246.0 & 146.5 & 8.4 \\
2 & Quat. & 147.2 & 231.8 & 137.4 & 72.4 \\
3 & $\mathrm{CH}$ & 103.1 & 176.6 & 124.5 & 8.2 \\
4 & Quat. & 123.8 & 201.0 & 172.1 & -1.8 \\
5 & Quat. & 123.5 & 217.2 & 169.0 & -15.8
\end{tabular}

High probability crystal structures were selected from among the 52 CSP candidates by calculating the agreement between theoretical and experimental ${ }^{13} \mathrm{C}$ shift tensors using an approach described elsewhere. ${ }^{[42]}$ The space groups $P 6 / \mathrm{mmm}$ and $P 31 \mathrm{~m}$ gave poor agreement with experimental data with rms errors of $\pm 8.3 \mathrm{ppm}$ and $\pm 8.7 \mathrm{ppm}$, respectively and were therefore rejected with high statistical confidence (i.e. 90\% confidence). The poor fit of these structures can be attributed to coulomb repulsion which reach a maximum when the layers are fully eclipsed. ${ }^{[21,23]}$ In the $P 31 m$ structure, further repulsion arises from the presence of four hydrogens which lie out of the ab-plane (see Figure 2). This unfavorable interaction results in the largest error found in any of the candidate structures evaluated.

Among the $\mathrm{Cmcm}$ and $P 21 / \mathrm{m}$ candidates, good agreement with experimental data was observed in 20 of the $23 \mathrm{Cmcm}$ cases with all 20 candidates having statistically equivalent fits at the $90 \%$ confidence level $(P=0.1)$. Likewise, 23 of the $26 P 21 / m$ structures exhibited good agreement to experiment and were therefore retained. The single best-fit $\mathrm{Cmcm}$ and $P 21 / \mathrm{m}$ candidates had respective offsets of $2.4 \AA$ and $2.2 \AA$. However, in both cases the region near the minimum is very broad with numerus candidates having agreement close to the best fit. As a result, the offsets identified from energy considerations of $1.4 \AA^{[21]}$ and $1.6 \AA^{[23]}$ lie within the 
error of the NMR best-fit structure. The NMR-based rejection of candidates that remain close to the original eclipsed configuration (i.e. $\mathrm{P} 6 / \mathrm{mmm}$ and $\mathrm{P} 31 \mathrm{~m}$ ) is consistent with the energy considerations discussed above showing that a slight offset between adjacent layers of COF-5 is energetically favorable due to a decrease in the coulombic repulsion. ${ }^{[21,23]}$

A single $C 2 / m$ candidate structure was also considered and the predicted NMR data found to be in close agreement with experimental values with an error of $6.0 \mathrm{ppm}$. This uncertainty is statistically indistinguishable from that observed in the $\mathrm{Cmcm}$ and $\mathrm{P} 21 / \mathrm{m}$ structures. Intriguingly, the $\mathrm{C} 2 / \mathrm{m}$ structure contains hydrogens that lie out of the ab plane, a feature found to be detrimental in the P31m model. However, the arrangement of the adjacent layers in the $C 2 / \mathrm{m}$ structure have an offset, apparently alleviating the columbic repulsion. We note that additional candidates for $\mathrm{C} 2 / \mathrm{m}$ are possible involving other offset distances and alternative hydrogen positions but were not considered in this initial study.

As described above, the NMR agreement of the candidate structures varies considerably for different space groups and even candidates within the same space group. In contrast, the PXRD patterns for all 52 candidate CSP structures shows almost no variation. Figure 5 illustrates the PXRD patterns from the five best-fit structures in each space group as selected by the NMR comparison. In all cases, only negligible differences are present. The NMR error for each CSP candidate is listed in Figure 5 to provide a convenient comparison of the PXRD and NMR methods. A more extensive comparison that includes all 52 CSP structures is given as Supporting Information and illustrates that all CSP candidates have nearly identical PXRD patterns. 


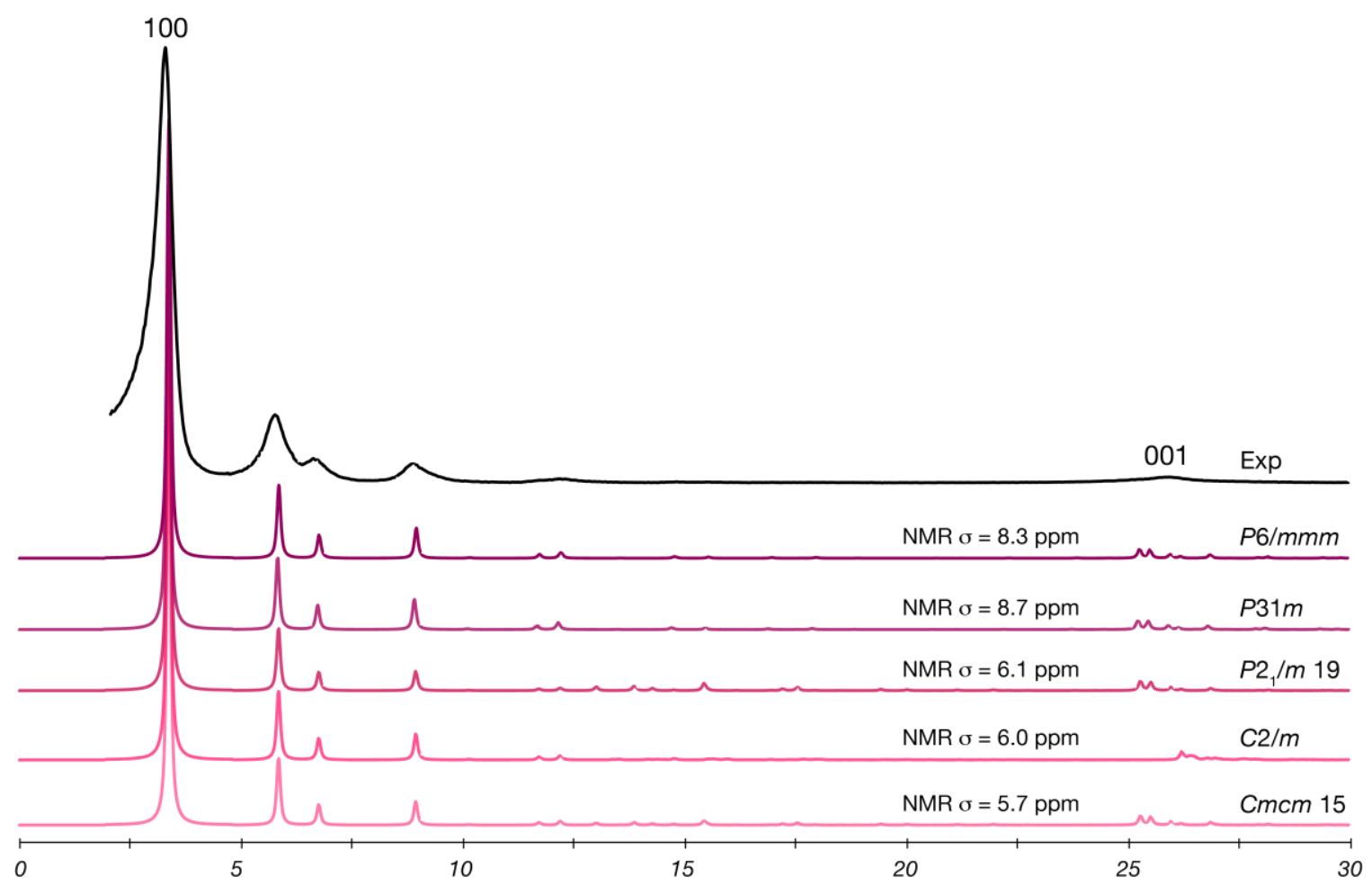

Figure 5. The experimental powder x-ray diffraction (PXRD) pattern for COF-5 (top) and the simulated PXRD patterns for the best-fit structures from the five space-groups considered herein. Additional CSP candidates were also retained and these have nearly identical XRPD patterns and are thus included as Supporting Information.

The SSNMR prediction that multiple space groups (i.e. $P 2_{1} / m, C m c m$, and $C 2 / m$ ) contribute to the overall structure of COF-5 supports the original contention of Yaghi that COF-5 is a material the contains turbostratic disorder. ${ }^{[1]}$ Our NMR analysis suggests that this disorder corresponds to the presence of three distinct space groups, all contained within a given stack of 2D layers and each corresponding to an offset of a particular symmetry. This assumption presumes that each space group is present in a sufficiently large quantity to influence the measured NMR tensors, which represent a weighted average of all the phases. This view is supported by the unusually wide lines in the ${ }^{13} \mathrm{C}$ isotropic spectrum where linewidths that are approximately five times wider than those obtained from microcrystalline pure phase materials 
on the same spectrometer. This is consistent with the presence of multiple phase all having slightly different chemical shifts and thus creating a broad line.

It is interesting to consider an alternative hypothesis for the structure of COF-5 in which the agreement to multiple space groups occurs because the powder consists of several distinct pure phases which are simply mixed together. Such mixed phase solids can form when the different phases have similar lattice energies. It is known that the NMR spectra of such solids usually exhibit at least some unique resonances for each phase and that the resulting spectrum is a superposition of the individual spectra. Such mixtures display linewidths similar to those observed in a pure phase material. An illustration such a mixture of two phases is given in Figure 6 for the 15 carbon flavonoid catechin ${ }^{[43,44]}$. In the case of COF-5, the absence of multiple resonances for each site argues that COF-5 is not a mixture of pure phase solids.

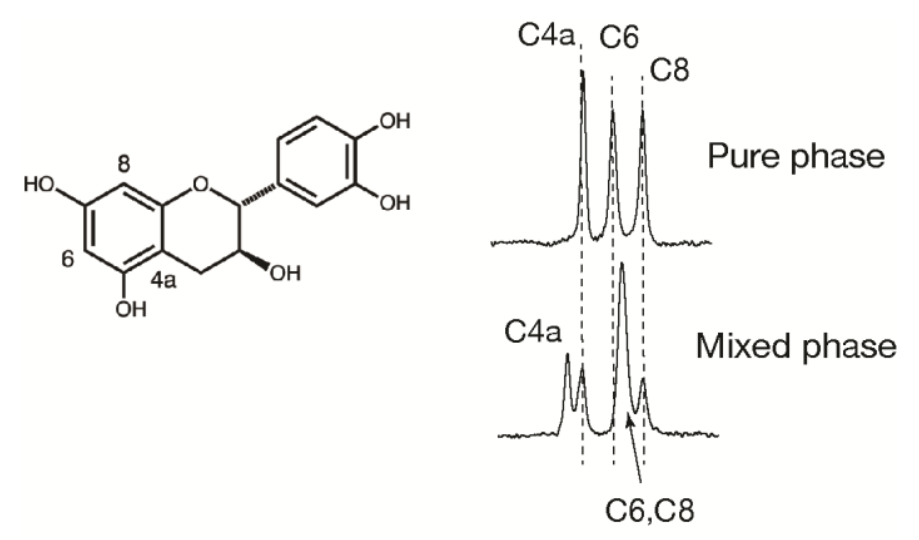

Figure 6. An illustration of a mixed phase material involving the flavonoid catechin (left). In the upper plot a region from the spectrum of the pure phase of catechin 4.5 hydrate is shown. The lower plot shows the development of a second phase consisting of catechin 4.5 hydrate plus catechin $\beta$-monohydrate that forms upon drying. The creation of new pure phases often creates new resonances for each site but does not change linewidths of either phase significantly. The absence of such new resonances in COF-5 is consistent with the conclusion that COF-5 is not a mixture of pure phase materials. 


\section{Conclusions.}

Prior studies have demonstrated that the $P 6 / \mathrm{mmm}$ space group originally proposed for COF-5 is inconsistent with energy considerations. ${ }^{[21,23]}$ The present study demonstrates that the $\mathrm{P6} / \mathrm{mmm}$ structure is also incompatible with ${ }^{13} \mathrm{C} \mathrm{NMR}$ shift tensor data. Alternative lattice structures are proposed in the space groups $\mathrm{Cmcm}, P 2_{1} / \mathrm{m}$ and $C 2 / m$, with numerous candidates having excellent agreement with both SSNMR, PXRD data and energy considerations. Because COF-5 is known to exhibit turbostratic disorder, it is posited that each of the three phases is, in fact, present within the powder and is therefore encoded in the experimental data. Evidence supporting this conclusion is found by comparing to recent ${ }^{13} \mathrm{C}$ benchmark data consisting of 309 tensor principal values which shows that the expected error for a pure phase material is approximately $\pm 3.3 \mathrm{ppm} .{ }^{[42]}$ In contrast the best fit candidate structures for COF-5 have errors of $\pm 5.7-6.0 \mathrm{ppm}$. This outcome is consistent with experimental ${ }^{13} \mathrm{C}$ tensor data that represent an average of differing principal values from three phases. Since the ${ }^{13} \mathrm{C}$ data is an average, none of the individual phases match exceptionally well, but numerous phases fit reasonably well.

Because COF-5 is the one of the first COFs to be characterized, subsequent work on COFs has often assumed that these materials primarily form with eclipsed stacking of layers. ${ }^{[21]}$ The present study suggests that COFs having eclipsed structures may be less common than expected and that a reassessment of 2D COF structures may prove beneficial.

\section{Experimental.}

Synthetic procedure for COF-5 was adapted from Côté et al. ${ }^{1}$ 
The FIREMAT ${ }^{13} \mathrm{C}$ solid-state NMR spectrum was acquired on a Chemagnetics CMX 200 spectrometer operating at $50.31 \mathrm{MHz}$ for ${ }^{13} \mathrm{C}, 200.04 \mathrm{MHz}$ for ${ }^{1} \mathrm{H}$ and using a PENCIL $5.0 \mathrm{~mm}$ probe. Decoupling was achieved with SPINAL-64 ${ }^{1} \mathrm{H}$ decoupling, a pulse duration of $8.9 \mu \mathrm{s}$ and standard phase angles. ${ }^{45}$ Acquisition parameters include ${ }^{1} \mathrm{H} 90^{\circ}$ and ${ }^{13} \mathrm{C} 180$ pulse durations of $3.9 \mu$ s and $8.8 \mu \mathrm{s}$, respectively, a cross-polarization time of $3.0 \mathrm{~ms}$, and a spinning rate of $661 \mathrm{~Hz}$. Spectral widths in the evolution and acquisition dimensions were $5.952 \mathrm{kHz}$ and $37.037 \mathrm{kHz}$, respectively. A total of 9 evolution increments were acquired and these were rearranged using a process described elsewhere ${ }^{[41]}$ to create 165 points. Data processing employed a process described elsewhere. ${ }^{[46]}$

All ${ }^{13} \mathrm{C}$ NMR chemical shieldings were calculated using the structures created by the crystal structure prediction process described above. Lattice factors were included by utilizing the software CASTEP and employing employed the GIPAW approach. ${ }^{[47,48]}$ The PW91 functional was employed with a plane-wave cutoff energy of $570 \mathrm{eV}$ and a K-point spacing of $0.07 \AA$. Shieldings were converted to chemical shifts using the relationship as described Klaus Eichele. ${ }^{[49]}$

Acknowledgements. This work was supported by the National Science Foundation under grant no. CHE-2016185 to J.K.H.

\section{Literature references.}

1. A. P. Côte, A. I. Benin, N. W. Ockwig, M. O’Keeffe, A. J. Matzger, O. M. Yaghi, Science 2005, 310, 1166.

2. Yaghi, O. M. ACS Cent. Sci. 2019, 5, 1295-1300. 
3. Sharma, R. K.; Yadav, P.; Yadav, M.; Gupta, R.; Rana, P.; Srivastana, A.; Zboril, R.; Varma, R. S.; Antonietti, M.; Gawande, M. B. Mater. Horiz. 2020, 7, 411 - 454.

4. Ding, S. -Y.; Wang, W. Chem. Soc. Rev. 2013, 42, $548-568$.

5. Wan, S.; Guo, J.; Kim, J.; Ihee, H.; Jiang, D. Angew. Chem. Int. Ed. 2008, 47, 8826-8830.

6. Wan, S.; Guo, J.; Kim, J.; Ihee, H.; Jiang, D. Angew. Chem. Int. Ed. 2009, 48, 5439 - 5442.

7. Sun, Q.; Aguila, B.; Perman, J.; Nguyen, N.; Ma, S. J. Am. Chem. Soc. 2016, 138, $15790-15796$.

8. Wang, H.; Wang, H.; Wang, Z.; Tang, L.; Zeng, G.; Xu, P.; Chen, M.; Xiong, T.; Zhou, C.; Li, X.; Huang, D.; Zhu, Y.; Wang, Z.; Tang, J. Chem. Soc. Rev. 2020, 49, 4135-4165.

9. Xiang, Z.; Xue, Y.; Cao, D.; Huang, L.; Chen. J. -F.; Dai, L. Angew. Chem. Int. Ed. 2014, 53, 2433 $-2437$.

10. Zhi, Y.; Shao, P.; Feng, X.; Xia, H.; Zhang, Y.; Shi, Z.; Mu, Y.; Liu, X. Mater. Chem. A 2018, 6, $374-382$.

11. Ma, D.; Wang, Y.; Liuy, A.; Li, S.; Chen, C. Catalysts, 2018, 8, 404.

12. Xu, H.; Chen, X.; Gao, J.; Lin, J; Addicoat, M.; Irle, S.; Jiang, D. Chem. Commun. 2014, 50, 12921294.

13. Fu, Y.; Zhu, X.; Huang, L.; Zhang, X.; Zhang, F.; Zhu, W. Appl. Catal. B 2018, 239, 46-51.

14. Zhang, F. -M.; Sheng, J. -L.; Yang, Z. -D.; Sun, X. -J.;Tang, H -L.; Lu, M.; Dong, H.; Shen, F. C.; Liu, J.; Lan, Y. -Q. Angew. Chem. Int. Ed. 2018, 130, $12282-12286$.

15. Zhang, C.; Zhang, S.; Yan, Y.; Xia, F.; Huang, A.; Xian, Y. ACS Appl. Mater. Interfaces 2017, 9, $13415-13421$.

16. Li, Z.; Zhang, Y.; Xia, H.; Mu, Y.; Liu, X Chem Commun. 2016, 52, 6613 - 6616. 
17. Yuan, S.; Li, X.; Zhu, J.; Zhang, G.; Puyvelde, P-V.; Van der Bruggen, B. Chem. Soc. Rev. 2019, 48, 2665-2681.

18. Bai, L.; Phua, S. Z. P.; Lim, W. Q.; Jana, A.; Luo, Z.; Tham, H. P.; Zhao, L.; Gao, Q.; Zhao, Y. Chem. Commun. 2016, 52, 4128-4131.

19. Furukawa, H.; Yaghi, O. M. J. Am. Chem. Soc. 2009, 131, 8875-8883.

20. Liu, Y.; Liu, D.; Yang, Q.; Zhong, C.; Mi, J. Ind. Eng. Chem. Res. 2010, 49, 2902-2906.

21. Lukose, B.; Kuc, A. Heine, T. Chem. Eur. J. 2011, 17, 2388.

22. Lukose, B.; Kuc, A.; Frenzel, J.; Heine, T. Beilstein J. Nanotechnol. 2010, 1, 60.

23. Koo, B. T.; Dichtel, W. R.; Clancy, P. J. Mater. Chem. 2012, 22, 17460.

24. Zhou, W.; Wu, H.; Yildrim, T. Chem. Phys. Lett. 2010, 499, 103.

25. Klapper, H.; Hahn, T. (2006) Point-group symmetry and physical properties of crystals. In: Hahn T. (eds) International Tables for Crystallography Volume A: Space-group symmetry. International Tables for Crystallography, vol A. Springer, Dordrecht.

26. Bhagavantam, S. Crystal Symmetry and Physical Properties, Academic press, New York, 1966.

27. Harper, J. K.; Grant, D. M. J. Am. Chem. Soc. 2000, 122, 3708-3714.

28. Harper, J. K.; Mulgrew, A. E.; Li, J. Y.; Barich, D. H.; Strobel, G. A.; Grant, D. M. J. Am. Chem. Soc. 2001, 123, 9837-9842.

29. Harper, J. K.; Barich, D. H.; Hu, J. Z.; Strobel, G. A.; Grant, D. M. J. Org. Chem. 2003, 68, 46094614.

30. Edgar, M.; Carter, V. J.; Tunstall, D. P.; Grewal, P.; Favre-Nicolin, V.; Cox, P. A.; Lightfoot, P.; Wright, P. A. Chem. Commun. 2002, 808-809.

31. Neumann, M. J. Chem. Phys. 1998, 109, 7300. 
32. Benedict, H.; Limbach, H. -H.; Wehln, M. J. Am. Chem. Soc. 1998, 120, 2939-2950.

33. Wang, L.; Uribe-Romo, F.; Mueller, L. J.; Harper, J. K. Phys. Chem. Chem. Phys. 2018, 20, 8475.

34. Baias, M.; Dumez, J. -N.; Svensson, P. H.; Schantz, S.; Day, G. M.; Emsley, L. J. Am. Chem. Soc. 2013, 135, 17501.

35. Kalakewich, K.; Iuliucci, R.; Harper, J. K. Cryst. Growth Des. 2013, 13, 5391.

36. Reilly, A. M.; Cooper, R. I.; Adjiman, C. S.; Bhattacharya, S.; Boese, A. D.; Brandenburg, J. G.; Bygrave, P. J.; Blysma, R.; Campbell, J. E.; Car, R.; Case, D. H.; Chadha, R.; Cole, J. C.; Cosburn, K.; Cuppen, H. M.; Curtis, F.; Day, G. M.; DiStasio Jr., R. A.; Dzyabchenko, A.; van Eijck, B. P.; Elking, D. M.; van den Ende, J. A.; Facelli, J. C.; Ferraro, M. B.; Fusti-Molnar, L.; Gatsiou, C. -A.; Gee, T. S.; de Gelder, R.; Ghiringhelli, L. M.; Goto, H.; Grimme, S.; Guo, R.; Hofmann, D. W. M.; Hoja, J.; Hylton, R. K.; Iuzzolino, L.; Jankiewicz, W.; de Jong, D. T.; Kendrick, J.; de Klerk, N. J. J.; Ko, H. -Y.; Kuleshova, L. N.; Li, X.; Lohani, S.; Leusen, F. J. J.; Lund, A. M.; Lv, J.; Ma, Y.; Marom, N.; Masunov, A. E.; McCabe, P.; McMahon, D. P.; Meekes, H.; Metz, M. P.; Misquitta, A. J.; Mohamad, S.; Monserrat, B.; Needs, R. J.; Nuemann, M. A.; Nyman, J.; Obata, S.; Oberhofer, H.; Oganov, A. R.; Orendt, A. M.; Pagola, G. I.; Pantelides, C. C.; Pickard, C. J.; Podeszwa, R.; Price, L. S.; Price, S. L.; Pulido, A.; Read, M. G.; Reuter, K.; Schneider, E.; Schober, C.; Shields, G. P.; Singh, P.; Sugden, I. J.; Szalewicz, K.; Taylor, C. R.; Tkatchenko, A.; Tuckerman, M. E.; Vacarro, F.; Vasileiadis, M.; Vazquez-Mayagoitia, A.; Vogt, L.; Wang, Y.; Watson, R. E.; de Wijs, G. A.; Yang, J.; Zhu, Q.; Groom, C. R. Acta Crystallogr., Sect. B: Struct. Sci., Cryst. Eng. Mater. 2016, 72, 439.

37. Dutour, J.; Guillou, N.; Huguenard, C.; Taulelle, F.; Mellot-Draznieks, C.; Ferey, G. Solid State Sci. 2004, 6, 1059. 
38. Taulelle, F.; Huguenard, C. Stud. Surf. Sci. Catal., 2001, 135, 163.

39. Opella, S. J.; Frey, M. H. J. Am. Chem. Soc. 1979, 101, 5854.

40. Alemany, L. B.; Grant, D. M.; Alger, T. D.; Pugmire, R. J. J. Am. Chem. Soc. 1983, 105, 6697.

41. Alderman, D. W.; McGeorge, G.; Hu, J. Z.; Pugmire, R. J.; Grant, D. M. Mol. Phys., 1998, 95, 1113-1126.

42. Holmes, S. T.; Engl, O. G.; Srnec, M. N.; Madura, J. D.; Quinones, R.; Harper, J. K.; Schurko, R. W.; Iuliucci, R. J. J. Phys. Chem. A 2020, 124, 3109.

43. Harper, J. K.; Doebbler, J. A.; Jacques, E.; Grant, D. M.; Von Dreele, R. B. J. Am. Chem. Soc. 2010, 132, 2928.

44. Harper, J. K.; Strohmeier, M.; Grant, D. M. J. Magn. Reson. 2007, 189, 20-31.

45. Fung, B. M.; Khitrin, A. K.; Ermolaev, K. J. Magn. Reson. 2000, 142, 97.

46. McGeorge, G.; Hu, J. Z.; Mayne, C. L.; Alderman, D. W.; Pugmire, R. J.; Grant, D. M. J. Magn. Reson. 1997, 129, 134.

47. Bonhomme, C.; Gervais, C.; Babonneau, F.; Coelho, C.; Pourpoint, F.; Azais, T.; Ashbrook, S. E.; Griffin, J. M.; Yates, J. R.; Mauri, F.; Pickard, C. J. First-principles calculation of NMR parameters using the gauge including projector augmented wave method: a chemist's point of view. Chem. Rev. 2012, 112, 5733-5779.

48. Pickard, C. J.; Mauri, F. All-electron magnetic response with pseudopotentials: NMR chemical shifts. Phys. Rev. B 2001, 63, 245101.

49. Eichele, K. NRM Ramblings Klaus Eichele. Anorganik.uni-tuebingen.de/Klaus/nmr/index.php?p=conventions/csa/csa (accessed January 17, 2019). 